\title{
Avaliação da qualidade de produtos contendo Maytenus ilicifolia Mart. ex Reissek - Celastraceae (espinheira-santa) comercializados na cidade de Umuarama - PR
}

\section{Quality evaluation of products with Maytenus ilicifolia Mart. ex Reissek - Celastraceae traded in the city of Umuarama - PR}

\author{
Ariadne Alvares Yokota ${ }^{1}$; Ezilda Jacomassi ${ }^{2}$; Antonio Laverde Junior ${ }^{3}$; Orlando \\ Seiko Takemura ${ }^{4}$
}

Resumo

\begin{abstract}
A Maytenus ilicifolia Mart. Ex Reissek (espinheira-santa), pertencente à família Celastraceae, é usada popularmente para tratamento de gastrite e de úlcera gástrica. O presente estudo teve como objetivo a análise da qualidade de produtos comercializados na cidade de Umuarama/PR, contendo M. ilicifolia. Para tanto, foram adquiridas 31 amostras de ervanários, farmácias de manipulação e dispensação, e supermercados. As amostras se apresentaram das seguintes formas: 11 na forma de cápsulas, 14 na forma de folhas íntegras, duas na forma de sachês, uma na forma de pó e três na forma de extrato fluido. As amostras foram analisadas sob vários aspectos: avaliação das embalagens, determinação de umidade, teor de cinzas totais, avaliação da pureza e análises cromatográficas. Os resultados obtidos na determinação de umidade mostraram que apenas 10,7\% das amostras apresentaram-se abaixo do valor permitido na determinação de água (máximo de 6\%) e 67,8\% das amostras atenderam o teor de cinzas totais (máximo de $8 \%$ ). Os resultados da cromatografia em camada delgada (CCD) mostraram que apenas $29 \%$ das amostras foram identificadas como M. ilicifolia, pela presença de catequinas e epicatequinas. Mais de 50\% das amostras analisadas apresentaram alto teor de impurezas. Concluindo, a maioria das amostras analisadas se mostrou inadequada ao consumo, seja pela presença de matéria orgânica estranha, teores de cinzas e umidade, além de as embalagens se apresentarem fora das especificações.

Palavras-chave: Maytenus ilicifolia. Cromatografia. Cinzas totais. Umidade. Catequinas. Epicatequinas.
\end{abstract}

\begin{abstract}
Maytenus ilicifolia Mart. Ex Reissek belongs to the Celastraceae family and is used for the treatment of gastritis and gastric ulcer. The purpose of this study was to analyze the quality of products containing Maytenus ilicifolia traded in the city of Umuarama - PR.. For this purpose, 31 of its samples were bought from herb dealers, pharmacies and supermarkets. Eleven samples were in capsule form, 14 'in natura', 2 in sachet form, one in powder form, and 3 in fluid extract form. The samples were analyzed considering several aspects: packaging evaluation, moisture content, total ashes content, purity evaluation and chromatographic analysis. Results obtained for moisture content showed that only $10.7 \%$ of the samples were below the allowed value concerning water content determination (limited at $6 \%$ ) and $67.8 \%$ were in accordance with the total ashes content (limited at $8 \%$ ). The thin layer chromatography (TLC) showed that only $29 \%$ of the samples were indentified as M. ilicifolia, due to the presence of catechins and epicatechins. Over $50 \%$ of the analyzed samples showed high content of impurities. In conclusion, most analyzed samples showed to be inappropriate for consumption, either for the presence of contaminants,
\end{abstract}

1 Acadêmica do Curso de Farmácia/UNIPAR.

2 Professora de Botânica Aplicada à Farmácia e Coordenadora do Horto Medicinal da UNIPAR.

3 Professor de Química Orgânica/UNIPAR.

${ }^{4}$ Farmacêutico e Biólogo formado pela Universidade Estadual de Londrina, Mestre em Ciências Biológicas pela Universidade Federal do Paraná, Doutor em Ciências Médicas pela Universidade de Gifu - Japão. E-mail: takemura@unipar.br 
or for the ashes and moisture contents, besides inappropriate packaging.

Key-words: Maytenus ilicifolia. Chromatography. Total ashes. Moisture. Catechins. Epicatechins.

\section{Introdução}

A espinheira-santa (Maytenus ilicifolia Mart. ex Reissek), também conhecida como cancerosa, cancrosa, espinheira-divina, espinho-de-deus, pertence à família Celastraceae. Trata-se de uma árvore de pequeno porte, nativa do Brasil, e tem como habitat natural, locais sombreados em regiões subtropicais e temperadas (CORREIA JUNIOR; MING; SCHEFER, 1991). As folhas secas são inodoras e com sabor suave, levemente adstringente (FARMACOPÉIA BRASILEIRA, 2004).

O efeito protetor da "espinheira-santa" é comparado ao da cimetidina, utilizada como antihistamínico de ação inibitória sobre a hemorragia digestiva e sobre a secreção gástrica de ácidos, comumente observados na úlcera péptica (MACAUBAS et al., 1988). Maytenus ilicifolia demonstrou, em ensaios pré-clínicos, uma ação potente contra úlcera gástrica (CARLINI; BRAZ, 1988; SOUZA-FORMIGONI et al., 1991). O extrato aquoso das folhas dessa espécie apresentou efeitos antiúlcerogênicos em pacientes portadores de dispepsia alta ou úlcera péptica (ALBERTON et al., 2002).

Estudos utilizando a cromatografia líquida de alta eficiência (CLAE) revelaram a presença de triterpenos e substâncias fenólicas em extratos brutos e hidroalcoólicos de $M$. ilicifolia e $M$. aquifolium Mart. (VILEGAS; LANÇAS; CERVI, 1994).

Maytenus ilicifolia encontra-se no mercado em diversas formas farmacêuticas, como tintura, extrato liofilizado, cápsulas e folhas íntegras, para uso na forma de chá (CÍRIO et al., 2003). A coleta indiscriminada de grandes quantidades de folhas, sem qualquer critério técnico, além de exterminar o patrimônio genético vegetal, tem levado à redução da ocorrência natural desta espécie (RADOMSKI et al., 2004).
Pela semelhança morfológica, espécies como a Sorocea bonplandii (Baill.) W.C. Burger, Lanj. \& Wess. Boer (Moraceae) e Zollernia ilicifolia (Brongn.) Vogel (Fabaceae), vêm sendo comercializadas como espinheira-santa $(M$. ilicifolia) (ALBERTON et al., 2002; JACOMASSI; MACHADO, 2003).

Embora se tenha parâmetro específico para a produção e comércio de fitoterápicos, a fraude e a má qualidade têm preocupado profissionais da área de saúde e também a comunidade científica. A ausência de qualidade, a adulteração e a utilização incorreta podem interferir na eficácia e até mesmo na segurança do uso do produto (MELO et al., 2004).

Sendo assim, o presente estudo objetivou a análise da qualidade de produtos comercializados na cidade de Umuarama/PR, contendo M. ilicifolia.

\section{Material e Métodos}

\section{Material vegetal}

Os materiais analisados nesta pesquisa consistiram de produtos à base de espinheira-santa oriunda de diversas empresas, comercializados na forma de cápsulas, folhas íntegras, sachês, planta pulverizada e extrato fluido. As amostras foram adquiridas em farmácias de dispensação, farmácias de manipulação, supermercados e ervanários da região de Umuarama, PR. Trinta e uma amostras foram avaliadas: 11 na forma de cápsulas, 14 folhas íntegras, duas na forma de saches, e uma amostra na forma de pó da planta, além de três produtos na forma de extrato fluido.

\section{Avaliação preliminar e preparação do material}

Inicialmente, informações de cada amostra analisada foram anotadas e, para cada produto, foi adotado um código utilizado ao longo de todo o experimento. $\mathrm{O}$ 
peso/volume real de cada amostra foi determinado e comparado com as informações da embalagem.

Alíquotas das plantas na forma de folhas íntegras foram pulverizadas em triturador industrial para a posterior realização dos ensaios de determinação de cinzas totais e teor de umidade.

As análises foram feitas em triplicata e foram apresentadas em média \pm DP (desvio-padrão).

\section{Avaliação das informações das embalagens}

A apresentação dos produtos e os pormenores de sua rotulagem foram avaliados, orientando-se pelos dados gerais da legislação para esse componente dos produtos. Verificou-se a presença e adequação dos itens: fornecedor, nome comercial, embalagem, bula, nome científico, instrução de uso, validade, peso, farmacêutico responsável, Conselho Regional de Farmácia (CRF), Cadastro Nacional de Pessoas Jurídicas (CNPJ), código de barra, selo e lacre, lote, endereço completo, gênero, espécie, conteúdo, logomarca, medicamento fitoterápico, uso adulto e/ ou pediátrico, indústria brasileira, sigla e número de registro no M.S., SAC, composição qualitativa e quantitativa, itens informativos: todo medicamento deve ser mantido fora do alcance de criança, conservar em temperatura ambiente, siga corretamente o modo de usar (NARITA et al., 2003).

\section{Determinação do teor de umidade por perda por dessecação}

As amostras foram submetidas à determinação da umidade em estufa comum. Amostras em triplicata dos materiais foram pesadas $(2 \mathrm{~g})$ em cápsulas de porcelana e colocadas em uma estufa pré-aquecida na temperatura de $105^{\circ} \mathrm{C}$, e nela permaneceram por 2 horas. Logo após, foram resfriados em dessecador e feita a primeira pesagem. Em seguida, o material foi colocado novamente na estufa, onde permaneceu por mais $2 \mathrm{~h}$. Logo em seguida, foi realizada uma nova pesagem, e assim sucessivamente, até a obtenção de peso constante, ou com diferença menor que $5 \mathrm{mg}$ (FARMACOPÉIA BRASILEIRA, 1998).

\section{Avaliação por cromatografia de camada delgada (ccd)}

As amostras foram analisadas em placas de gel de sílica 60 F254, e utilizou-se acetato de etila, ácido fórmico e água (95: 5: 5), como fase móvel. Como revelador, foi utilizada a vanilina sulfúrica (FARMACOPÉIA BRASILEIRA, 2004).

\section{Determinação de elementos estranhos}

Na determinação de matérias orgânicas estranhas, foram separados materiais estranhos (pedaços de caule que estavam junto com as folhas), pesados e anotados seus valores. Foram registradas as alíquotas e calculadas as percentagens da matéria orgânica estranha.

\section{Determinação do teor de cinzas totais}

$\mathrm{Na}$ determinação de cinzas, foram pesados 2,0 $\mathrm{g}$ das amostras de folhas íntegras pulverizadas e em cápsula em um cadinho previamente calcinado. Após a distribuição da amostra no cadinho, foi procedida à incineração do material em mufla com temperatura não superior a $450^{\circ} \mathrm{C}$. Em seguida, efetuou-se o cálculo de porcentagem de cinzas (FARMACOPÉIA BRASILEIRA, 1998).

\section{Resultados e discussão}

Na Tabela 1, podem ser observados os resultados da análise relacionados à adequação de rotulagem, e presença dos itens e dados na embalagem. Dentre as amostras analisadas, todas indicavam o peso e o nome comercial, porém apenas três amostras (15\%) apresentavam bula, sete constavam o nome científico da planta (35\%), 13 (65\%) traziam as instruções de uso. As amostras adquiridas em farmácias de manipulação não foram avaliadas com relação aos componentes de 
rótulos, por seguir legislação própria. As ausências de bula e de informações na embalagem dificultam o acesso a informações importantes acerca do

Tabela 1. Adequação dos itens de identificação e rotulagem das amostras de Maytenus ilicifolia Mart. ex Reissek Celastraceae (espinheira-santa) comercializadas na cidade de Umuarama - PR.

\begin{tabular}{|c|c|c|}
\hline ITENS & OSTRAS & PERCENTUAL(\%) \\
\hline Fornecedor & 19 & 95 \\
\hline Nome Comercial & 20 & 100 \\
\hline Embalagem & 20 & 100 \\
\hline Bula & 3 & 15 \\
\hline Nome Científico & 7 & 35 \\
\hline Instrução de uso & 13 & 65 \\
\hline Validade & 13 & 65 \\
\hline Farmacêutico Responsável (CRF) & 16 & 80 \\
\hline CNPJ & 18 & 90 \\
\hline Código de barras & 11 & 55 \\
\hline Selo-lacre & 15 & 75 \\
\hline Lote & 18 & 90 \\
\hline Endereço completo & 14 & 70 \\
\hline Gênero & 7 & 35 \\
\hline Espécie & 7 & 35 \\
\hline Conteúdo & 14 & 70 \\
\hline Logomarca & 13 & 65 \\
\hline Medicamento Fitoterápico & 3 & 15 \\
\hline Uso (adulto e/ou pediátrico) & 2 & 7 \\
\hline Indústria brasileira & 3 & 15 \\
\hline Sigla e número de registro no M.S. & 5 & 25 \\
\hline SAC & 7 & 35 \\
\hline Composição (qualitativa e quantitativa) & 9 & 45 \\
\hline Advertência* & 4 & 20 \\
\hline Advertência** & 7 & 35 \\
\hline Advertência*** & 4 & 20 \\
\hline Peso & 20 & 100 \\
\hline
\end{tabular}

Advertência*: todo medicamento deve ser mantido fora do alcance de crianças; Advertência**: Conservar o produto em temperatura ambiente e protegido da luz e umidade; Advertência***: Siga corretamente o modo de usar. Não desaparecendo os sintomas, procure orientação médica. 
Dentre as amostras analisadas (cápsulas e sachês), com relação ao peso especificado na embalagem, os resultados indicaram que $23 \%$ das cápsulas analisadas apresentaram peso inferior ao especificado (Tabela 2). Algumas das amostras analisadas apresentaram peso superior ao que era indicado na embalagem. A amostra C7 ultrapassou em mais de $50 \%$ o peso especificado e não continha na sua bula outros componentes anunciados. Além de se constituir uma fraude ao consumidor, diferenças entre o peso especificado real e o peso declarado podem significar ausência ou ineficiência dos testes de controle de qualidade na empresa.

Tabela 2. Avaliação do peso das amostras de Maytenus ilicifolia Mart. ex Reissek - Celastraceae (espinheira-santa) comercializadas na cidade de Umuarama - PR

\begin{tabular}{llll}
\hline AMOSTRA & Peso Esperado $(\mathbf{m g})$ & MEDIA(mg) & DP \\
\hline C1 & 270 & 242,0 & 7,3 \\
C2 & 300 & 311,3 & 44,3 \\
C3 & 360 & 385,9 & 15,3 \\
C4 & 380 & 420,8 & 12,0 \\
C5 & 350 & 352,3 & 11,8 \\
C6 & 380 & 406,1 & 11,7 \\
C7 & 360 & 575,2 & 13,2 \\
C8 & 200 & 188,6 & 6,9 \\
C9 & 300 & 235,8 & 9,9 \\
C10 & 400 & 395,6 & 10,8 \\
C11 & 300 & 296,8 & 9,1 \\
S1 & 1.000 & $1.081,4$ & 44,4 \\
S2 & 1.000 & $1.084,6$ & 55,3 \\
\hline
\end{tabular}

C: cápsula; S: sachê. DP: Desvio padrão.

Analisando os resultados obtidos nos ensaios (Tabela 3), apenas três das amostras apresentaram valores permitidos com relação aos limites de teores de umidade para a planta, e dezenove amostras para cinzas totais. Segundo a Farmacopéia Brasileira (2004), o limite permitido para umidade é de no máximo $6 \%$, e, para cinzas totais, no máximo $8 \%$. Teores elevados de cinzas podem revelar a presença de elementos minerais como terra ou areia, indício de possíveis contaminações, e enquanto teores elevados de umidade podem significar tanto procedimentos de estabilização ou armazenamento inadequados. Altos teores de umidade podem favorecer tanto a ação enzimática e a proliferação microbiana, o que pode comprometer a qualidade das amostras (AMARAL et al., 2003). 
Tabela 3. Avaliação dos teores de umidade e cinza, presentes nas amostras de Maytenus ilicifolia Mart. ex Reissek Celastraceae (espinheira-santa) comercializadas na cidade de Umuarama - PR.

\begin{tabular}{|c|c|c|}
\hline AMOSTRA & UMIDADE (média \pm DP) & CINZA(média $\pm \mathrm{DP}$ ) \\
\hline $\mathrm{C} 1$ & $7,1 \pm 0,2$ & $9,3 \pm 0,09$ \\
\hline $\mathrm{C} 2$ & $8,6 \pm 0,5$ & $8,9 \pm 0,23$ \\
\hline $\mathrm{C} 3$ & $6,6 \pm 0,3$ & $6,0 \pm 0,19$ \\
\hline $\mathrm{C} 4$ & $11,0 \pm 0,1$ & $2,9 \pm 0,04$ \\
\hline $\mathrm{C} 5$ & $5,7 \pm 0,1$ & $8,4 \pm 0,04$ \\
\hline C6 & $10,0 \pm 0,5$ & $1,9 \pm 0,01$ \\
\hline $\mathrm{C} 7$ & $8,0 \pm 6,4$ & $5,4 \pm 0,06$ \\
\hline $\mathrm{C} 8$ & $9,9 \pm 0,6$ & $8,1 \pm 0,06$ \\
\hline C9 & $7,8 \pm 0,5$ & $9,7 \pm 0,03$ \\
\hline $\mathrm{C} 10$ & $6,8 \pm 0,6$ & $5,6 \pm 0,02$ \\
\hline $\mathrm{C} 11$ & $7,9 \pm 0,5$ & $8,5 \pm 0,04$ \\
\hline $\mathrm{F} 1$ & $8,9 \pm 0,5$ & $9,8 \pm 0,03$ \\
\hline F2 & $8,9 \pm 0,3$ & $9,4 \pm 0,07$ \\
\hline F3 & ND & $9,4 \pm 0,03$ \\
\hline F4 & $7,9 \pm 0,8$ & $5,4 \pm 0,08$ \\
\hline F5 & $9,6 \pm 0,4$ & $5,3 \pm 0,03$ \\
\hline F6 & $8,5 \pm 1,4$ & $12,9 \pm 0,14$ \\
\hline F7 & $10,0 \pm 4,5$ & $9,4 \pm 0,02$ \\
\hline F8 & $9,7 \pm 0,4$ & $5,8 \pm 0,00$ \\
\hline F9 & $9,7 \pm 0,3$ & $6,9 \pm 0,01$ \\
\hline F10 & $9,2 \pm 0,1$ & $2,3 \pm 0,03$ \\
\hline F11 & $10,8 \pm 0,1$ & $1,9 \pm 0,02$ \\
\hline F12 & $8,4 \pm 0,4$ & $6,8 \pm 0,03$ \\
\hline F13 & $9,5 \pm 0,2$ & $9,3 \pm 0,04$ \\
\hline F14 & $10,8 \pm 3,7$ & $5,4 \pm 0,02$ \\
\hline S1 & $15,9 \pm 9,7$ & $6,9 \pm 0,05$ \\
\hline $\mathrm{S} 2$ & $10,0 \pm 0,8$ & $7,2 \pm 0,05$ \\
\hline P1 & $8,7 \pm 0,3$ & $8,7 \pm 0,08$ \\
\hline
\end{tabular}


Quando analisado o requisito elementos das plantas, devem-se possivelmente a problemas estranhos, apenas cinco amostras dentre as 14 de de manejo, limpeza e separação inadequada de folhas íntegras (F2, F5, F6, F7, F14) foram aprovadas (Tabela 4). Entre as nove amostras reprovadas, os índices superaram o limite permitido pela Farmacopéia Brasileira (2004), que é de no máximo $2 \%$, para a maioria das drogas vegetais (MELO et al., 2004). Os índices altos de impurezas, para a maioria produtos à base de plantas medicinais, porém, para a espinheira-santa, o alto teor de impurezas devese principalmente a inclusão de caules. Em algumas amostras como F1, F3 e F4, o teor de impurezas (caules) ou a quantidade de folhas foi elevado.

Tabela 4. Porcentagem de impurezas presentes na amostra "in natura" de Maytenus ilicifolia Mart. ex Reissek Celastraceae (espinheira-santa) comercializadas na cidade de Umuarama - PR.

\begin{tabular}{ll}
\hline AMOSTRA & IMPUREZAS \\
\hline F1 & $51,10 \%$ \\
F2 & $0,09 \%$ \\
F3 & $71,90 \%$ \\
F4 & $76,70 \%$ \\
F5 & $0,98 \%$ \\
F6 & $0,68 \%$ \\
F7 & $2,15 \%$ \\
F8 & $31,80 \%$ \\
F9 & $8,70 \%$ \\
F10 & $42,00 \%$ \\
F11 & $44,64 \%$ \\
F12 & $34,25 \%$ \\
F13 & $9,66 \%$ \\
F14 & $1,50 \%$ \\
\hline
\end{tabular}

Dentre as amostras analisadas, apenas 29\% do total apresentaram identificação positiva para M. ilicifolia referente à análise cromatográfica. A identificação foi feita pela verificação da presença de epicatequina $\mathrm{Rf}$ aproximadamente 0,50, 0,60, 0,70 catequina, Rf de aproximadamente 0,80
(FARMACOPÉIA BRASILEIRA, 2004), porém, $46,4 \%(n=13)$ das amostras apresentaram uma substância não identificada entre $\mathrm{Rf}=0,89$ a 0,92 (Tabelas 5 e 6). Essa substância apresentava a mesma coloração bordô apresentada pelas catequinas, após a revelação com vanilina sulfúrica. 
Tabela 5. Valores de Rf de substâncias presentes nas amostras (folhas "in natura" e extrato fluido) de Maytenus ilicifolia Mart. ex Reissek - Celastraceae (espinheira-santa) comercializadas na cidade de Umuarama - PR.

\begin{tabular}{lccccc}
\hline AMOSTRA & MANCHA1 (RF) & MANCHA 2 (RF) & MANCHA3(RF) & MANCHA 4(RF) & MANCHA 5(RF) \\
\hline P & 0,59 & 0,68 & 0,73 & 0,82 & 0,91 \\
F1 & - & 0,68 & 0,74 & 0,81 & 0,92 \\
F2 & - & - & - & 0,81 & - \\
F4 & - & 0,67 & 0,73 & 0,81 & 0,92 \\
F8 & - & - & - & 0,81 & - \\
F10 & 0,57 & 0,67 & 0,73 & 0,81 & 0,89 \\
F12 & 0,56 & 0,68 & - & 0,82 & 0,92 \\
P & 0,59 & 0,68 & 0,73 & 0,82 & 0,92 \\
E.F. & 0,58 & 0,69 & 0,74 & 0,82 & 0,92 \\
\hline
\end{tabular}

P: padrão; F: folha; E.F.: extrato fluido.

Tabela 6. Valores de Rf de substâncias presentes nas amostras de Maytenus ilicifolia Mart. ex Reissek - Celastraceae (espinheira-santa) comercializadas na cidade de Umuarama - PR.

\begin{tabular}{lccccc}
\hline AMOSTRA MANCHA 1 (RF) & MANCHA 2 (RF) & MANCHA 3 (RF) & MANCHA 4 (RF) & MANCHA 5 (RF) \\
\hline P & 0,49 & 0,59 & 0,67 & 0,77 & 0,91 \\
C3 & - & 0,58 & 0,63 & 0,74 & 0,87 \\
C4 & - & - & - & 0,68 & - \\
C6 & - & 0,58 & 0,62 & 0,67 & 0,89 \\
C10 & - & 0,58 & 0,63 & 0,73 & 0,88 \\
P & 0,46 & 0,58 & 0,65 & 0,73 & 0,87 \\
S1 & - & - & 0,65 & 0,74 & - \\
P & 0,48 & 0,58 & 0,65 & 0,75 & 0,91 \\
S2 & - & - & 0,64 & 0,74 & - \\
\hline
\end{tabular}

P: padrão; C: cápsula; S: sache

Duas amostras de folhas íntegras, uma de

Os resultados obtidos sugerem que as amostras cápsulas, duas na forma de sachês, correspondendo a $18 \%$ das amostras, apresentaram somente traços de catequina, desprovidas de fluorescência a $365 \mathrm{~nm}$ e extinção de fluorescência a $254 \mathrm{~nm}$. No entanto, o padrão cromatográfico de $53 \%$ das amostras analisadas não foi compatível com a amostra padrão de M. ilicifolia. ora propostas como M. ilicifolia, as folhas íntegras F1, F4, F8, F10 e F12, apesar de apresentarem resultado de identidades positivas, possuem alto teor de impurezas (Tabela 4). As outras amostras analisadas não foram identificadas como $M$. ilicifolia. Espécies como Sorocea bomplandii ou Zollernia ilicifolia são espécies mais utilizadas para 
adulteração de espinheira-santa (ALBERTON et al., 2002).

O controle de qualidade envolvendo matériasprimas vegetais para elaboração de fitoterápicos é de extrema importância para garantir segurança e eficácia aos seus usuários. Entretanto, o aumento no número de medicamentos à base de plantas, disponíveis à população, não é proporcional à sua qualidade (FARIAS, 2001).

No Brasil, a regulamentação para a comercialização de fitoterápicos é regida por legislação específica, como portarias da Vigilância Sanitária e por monografias para espécies vegetais, que contêm critérios definidos para identidade, pureza e teor de constituintes químicos (MELO et al., 2004). No entanto, os mesmos autores ressaltam a precariedade no controle de qualidade de fitoterápicos, e afirmam a necessidade de uma forte intensificação da vigilância por parte dos órgãos competentes, para a obtenção de produtos seguros e eficazes. Segundo Nascimento et al. (2005), é imprescindível a existência de um controle de qualidade rígido e de fiscalização mais assídua por parte dos órgãos competentes.

\section{Conclusão}

A análise dos resultados obtidos no presente trabalho permite concluir que a maioria dos produtos contendo espinheira-santa comercializados na cidade de Umuarama - PR, não se encontra em conformidade com as exigências da legislação vigente. Altos índices de reprovação foram encontrados em relação a teores de umidade, presença de elementos estranhos e também nos testes de identidade (CCD). Irregularidades foram percebidas também na análise de informações de rótulos e presença de bulas.

Os resultados observados no trabalho mostram a necessidade de uma eficaz fiscalização de produtos comercializados por parte da Vigilância Sanitária, uma vez que produtos de má qualidade podem colocar em risco a saúde das pessoas que buscam a cura de doenças utilizando plantas medicinais.

\section{Referências}

ALBERTON, M. D.; SOUZA, E. S.; FALKENBERG, D. B.; FALKENBERG, M. B. Identificação de marcadores cromatográficos de Zollernia ilicifolia e Sorocea bonplandii para o controle de qualidade de espinheirasanta. Revista Brasileira Farmacognosia, João Pessoa, v. 12, p. 9-10, 2002.

AMARAL, F. M. M.; COUTINHO, D. F.; RIBEIRO, M. N. S.; OLIVEIRA, M. A. Avaliação da qualidade de drogas vegetais comercializadas em São Luís/Maranhão. Revista Brasileira de Farmacognosia, João Pessoa, v. 13, supl., p. 27-30, 2003.

CARLINI, E. L.A.; BRAZ, S. Efeito protetor do liofilizado obtido do abafado de Maytenus sp (espinheira-santa) contra úlcera gástrica experimental em ratos. In:

Estudo da ação antiúlcera gástrica de plantas brasileiras: Maytenus ilicifolia (espinheira-santa) e outras. Brasília: Central de Medicamentos, 1988. p. 21-35.

CÍRIO, G. M.; DONI FILHO, L.; MIGUEL, M. D.; MIGUEL, O. G.; ZANIN, S. M. W. Interrelação de parâmetros agronômicos e físicos de controle de qualidade de Maytenus ilicifolia, Mart. ex. Reiss (espinheirasanta) como insumo para a indústria farmacêutica. Visão acadêmica, Curitiba, v. 4, p. 67-76, 2003.

CORREIA JUNIOR, C.; MING, L. C.; SCHEFFER, M. C. Cultivo de plantas medicinais, condimentares $e$ aromáticas. Curitiba: EMATER, 1991.

FARIAS, M. R. Avaliação da qualidade de matériasprimas vegetais. In: SIMÔES, C. M. O. et al. Farmacognosia da planta ao medicamento. Santa Catarina: EDUFSC, 2001. p. 199-222.

FARMACOPÉIA BRASILEIRA. 4. ed. São Paulo: Atheneu, 1998. Parte 1.

FARMACOPÉIA BRASILEIRA. 4. ed. São Paulo: Atheneu, 2004. Fasc. 5.

JACOMASSI, E.; MACHADO, S. R. Características anatômicas de espinheira-santa (Maytenus ilicifolia Mart. ex. Reissek e Maytenus aquifolia Mart.) e mataolho (Sorocea bonplandii (Baill.) Burg. Lanj. \& Bôer.) para o controle de qualidade da matéria-prima. Revista Brasileira Plantas Medicinais, Botucatu, v. 6, p. 84-96, 2003.

MACAUBAS, C. I. P.; OLIVEIRA, M. G. M.; FORMIGONE, M. L. O.; SILVEIRA FILHO, N. G.; CARLINI, E. A. Estudo da eventual ação antiúlcera 
gástrica do bálsamo (Sedum sp), folha-da-fortuna (Bryofillum calycinum), couve (Brassica oleraceae) e da espinheira-santa ( Maytenus ilicifolia) em ratos. In: Estudo da ação antiúlcera gástrica de plantas brasileiras: Maytenus ilicifolia (espinheira-santa) e outras. Brasília: Central de Medicamentos, 1988. p. 5-20.

MELO, J. G.; NASCIMENTO, V. T.; AMORIM, E. L. C.; ANDRADE LIMA, C. S.; ALBUQUERQUE, U. P. Avaliação da qualidade de amostras comerciais de boldo (Peumus boldus Molina), pata-de-vaca (Bauhinia spp.) e ginco (Ginkgo biloba L.). Revista Brasileira Farmacognosia, João Pessoa, v. 14, n. 2, p. 111-120, 2004.

NARITA, E.; NEITZKE, H. C.; SOUZA, F. C. D.; MONTEIRO, L. P.; MARQUES, L. C. Controle de qualidade farmacobotânico de drogas vegetais comercializadas em Maringá - ano 2002. Infarma, Brasília, v. 15, p. 70-73, 2003.

NASCIMENTO, V. T.; LACERDA, E. U.; MELO, J. G.; LIMA, C. S. A; AMORIM, E. L. C.; ALBUQUERQUE, U. P. Controle de qualidade de produtos à base de plantas medicinais comercializados na cidade do RecifePE: erva-doce (Pimpinella anisum L.), quebra-pedra (Phyllanthus spp), espinheira-santa (Maytenus ilicifolia Mart.) e camomila (Matricaria recutita L.). Revista Brasileira de Plantas Medicinais, Botucatu, v. 7, n. 3, p. 56-64, 2005.

RADOMSKI, M. I.; WISNIEWSKI, C.; CURCIO, G. R.; RACHWAI, M. G.; SANTOS, C. A. M. Caracterização de ambientes de ocorrência natural e sua influência sobre o peso específico e o teor de polifenóis totais de folhas de espinheira-santa (Maytenus ilicifolia Mart.). Revista Brasileira Plantas Medicinais, Botucatu, v. 6, p. 36-43, 2004.

SILVA, T.; DAL-PIZZOL, F.; BELLO, C. M.; MENGUE, S. S.; SCHENKEL, E. P. Bulas de medicamentos e a informação adequada ao paciente. Revista de Saúde Pública, São Paulo, v. 34, p. 184-189, 2000.

SOUZA-FORMIGONE, M. L. O.; OLIVEIRA, M. G. M.; MONTEIRO, M. G.; SILVEIRA-FILHO, N. G.; BRAZ, S.; CARLINI, E. A. Antiulcerogenic effects of two Maytenus species in laboratory animals. Journal Ethnopharmacology, Lausanne, v. 34, p. 21-27, 1991.

VILEGAS, J. H. Y.; LANÇAS, F. M.; CERVI, A. C. High resolution gas chromatography analysis of "espinheirasanta" (Maytenus ilicifolia and Maytenus aquifolium): analysis of crude drog adulterations. Phytotherapy Research, New Jersey, v. 8, p. 241-244, 1994.

Recebido em: 26 de junho de 2008

Aceito em: 13 de agosto de 2010 